\title{
Designing a mobile-based self-care application for patients with cutaneous leishmaniasis: An effective step in patients' self-care and participation
}

\author{
Khadije Nadri ${ }^{1}$, Hadieh Sajedi ${ }^{*}$, Amin Sayahi ${ }^{3}$, Leila Shahmoradi ${ }^{4,5^{*}}$ \\ ${ }^{1} \mathrm{MSc}$, Department of Health Information Management, Faculty of Allied Medical Sciences, Tehran University of Medical Sciences, Tehran, Iran \\ ${ }^{2}$ Department of Computer Sciences, School of Mathematics, Statistics and Computer Sciences, College of Sciences, University of Tehran, \\ Tehran, Iran \\ ${ }^{3}$ Chakavak Company, Tehran, Iran \\ ${ }^{4}$ Halal Research Center of IRI, Food and Drug Administration, Tehran, Iran \\ ${ }^{5}$ Department of Health Information Management, Faculty of Allied Medical Sciences, Tehran University of Medical Sciences, Tehran, Iran
}

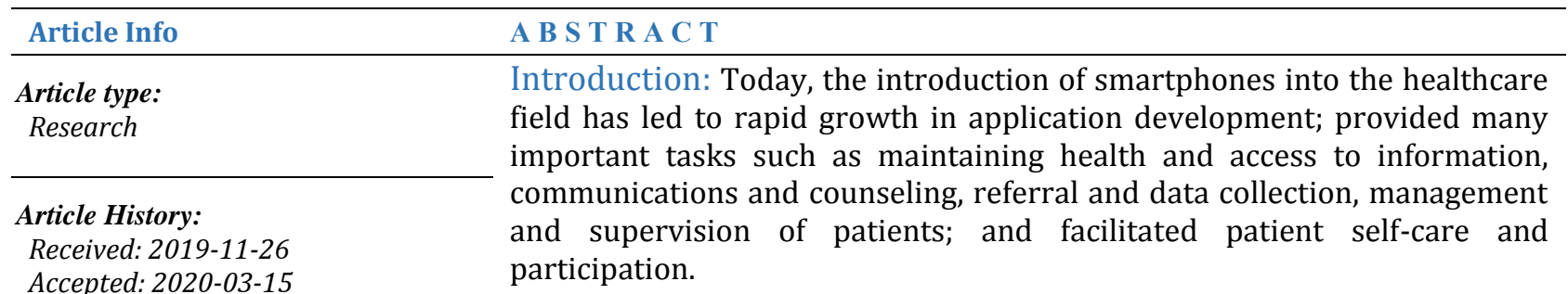

Published: 2020-03-21

Material and Methods: In order to design a mobile-based self-care

* Corresponding author:

Hadieh Sajedi

Department of Computer Sciences, School of Mathematics, Statistics and Computer Sciences, College of Sciences, University of Tehran, Tehran, Iran

Email: hhsajedi@ut.ac.ir

Leila Shahmoradi

Department of Health Information Management, Faculty of Allied Medical Sciences, Tehran University of Medical Sciences, Tehran, Iran

Email: Ishahmoradi@tums.ac.ir

\section{Keywords:}

Self-Care

Application

Leishmaniasis application, the designing purpose was first defined and then related literature was reviewed and different kinds of software designed in this field were identified. Using the specified information elements, UML diagrams (Use-Case, Sequence, and Activity) were plotted by Visual Paradigm Enterprise 8 software.

In fact, a feasibility assessment was carried out, based on which the application prototype was developed and provided to 10 patients to achieve their opinions. In the next step, the final application components were determined based on user feedback and experts approval. In the final step, Quiz Satisfaction Survey Questionnaire v.7.0 was used to assess users' satisfaction.

Results: Based on user questions and answers, and answers available in the database, the application offers appropriate therapies that emphasize the presence of patients in health centers.

Conclusion: The presented application helps with providing rapid information and awareness about different aspects of the disease.

Cite this paper as:

Nadri K, Sajedi H, Sayahi A, Shahmoradi L. Designing a Mobile-Based Self-Care Application for Patients with Cutaneous Leishmaniasis: An Effective Step in Patients' Self-Care and Participation. Front Health Inform. 2020; 9: 29. DOI: 10.30699/fhi.v9i1.210

\section{INTRODUCTION}

Mobile health (mHealth) refers to the use of mobile phones in the provision of health services to improve people's health [1]. mHealth is one of the subcategories of eHealth that provides a proper platform for healthcare development and management, ranging from access to services to education, and has changed the form of healthcare provision worldwide $[\underline{2}, \underline{3}]$. The number of $\mathrm{mHealth}$ apps by the end of 2017 was estimated at 325,000
[4]. The mHealth application market will grow by $15 \%$ to reach $\$ 31$ billion by 2020 [ㅁ] .

mHealth users can manage their information and communicate with specialist physicians to improve care quality and efficiency. With the growing number of mobile users and the expansion of smartphones and health apps, mHealth uses are on the rise. The advent of smartphone technology along with interactive displays has made it possible for patients and service providers to quickly and easily transmit and track information. Patients can better 
manage and control their illness and health using wireless technologies and developed software []․ mHealth uses can be divided into several main groups of education and empowerment, information gathering, remote monitoring and care, and service delivery coordination. Although mHealth has been employed in various diseases, its role in improving self-care has been greater. Mobile apps, as part of customer-centered health informatics for facilitating self-care processes, have the potential for patient education, disease management, follow-up, and motivation increase. mHealth systems allow for selfcare facilitation and improvement with the use of disease management software and provision of capabilities such as the ability to collect clinical symptoms and data for patients, management and tracking of pharmaceutical and therapeutic processes and lifestyle changes [7]. mHealth has the potential to be used as a low-cost, available way of complementing the provision of specialized services, especially in economically or geographically deprived areas, thereby filling the gap in gaining access to specialized services []ㅜ. The use of mHealth is especially effective in educating patients about self-care and preventing the spread of diseases [9]. There are many options for disease control, one of which is self-care. Self-care in patients with Cutaneous Leishmaniasis (CL) is very important. Effective self-care is an important factor in promoting positive health outcomes and preventing future complications [10]. Self-care content can be changed depending on the target group, their specific needs, and different ways of meeting the needs. For instance, a self-care application for skin diseases or hundreds of other topics can be designed employing a variety of ways or formats. With the transition of diseases from communicable to non-communicable and moving from definitive treatment to lifelong care, the role of people in self-care is increasing every day, estimated to account for $65-85 \%$ of all care. Self-care domains include health promotion, lifestyle modification, disease prevention, self-assessment [11], health maintenance, and participation in treatment. Employing mHealth emerged from the combination of smartphone technology and medical expertise, and provides new opportunities to improve patient health and their participation in disease management. Therefore, this study aimed to design a mobile-based self-care application as an effective step toward self-care and participation of CL patients.

CL is a potentially severe and disfiguring disease. People with CL have one or several long-lasting lesions on the skin, usually without fever or general symptoms. The impact that CL has on propagating poverty is important, since treatment is expensive and is therefore either unaffordable or involves a great loss of wages [12]. 'Health app' was defined as any commercially available health or fitness application with capacity for self-monitoring [13]. A growing number of studies have explored the impact of technological interventions on consumers' health outcomes. These interventions have included automated reminders (via text messaging) and internet-based information and have been assessed using self-report by participants with little, if any, external validation.

There are several mobile-based applications with the aim of self-caring. In the following, we mention to some of them. Design and evaluation of a mobilebased application for patients with type 2 Diabetes has been done in [14]. Taheri et al. developed a mobile-based self-care application for patients with breast cancer undergoing chemotherapy [15]. To best of our knowledge, for CL disease there are no self-caring application.

\section{MATERIAL AND METHODS}

In order to design a mobile-based self-care application, the designing purpose was first defined and then related literature was reviewed and different kinds of software designed in this field were identified. Using the specified information elements, UML diagrams (Use-Case, Sequence, and Activity) were plotted by Visual Paradigm Enterprise 8 software. Then the graphic parts of the application were designed using Photoshop and Jquery Mobile software.

Then the application environment was described and system analysis and design were carried out. In fact, a feasibility assessment was carried out, based on which the application prototype was developed and provided to 10 patients to achieve their opinions. In the next step, the final application components were determined based on user feedback and experts approval.

The application was programmed in Java in the Android studio-programming environment based on the following versions:

Android Studio 3.4, JRE:1.8.0_152-release-1343-b01 AMD 64, JVM: OpenJDK 64-Bit Server VM by JetBrains. The SQL Server database was used to store and retrieve information upon user request and the PHP programming language was used to link application activities (login, registration, forgot passwords, contact with physicians) to the database. That is, the application sends its request and after receiving information from the database, the categorized information is sent to the application. After installing the application, users first enter their username and password and then enter the application home screen. The application's main menu includes User Information, Disease Information, Education and Prevention, Contact with Physicians and Useful Links. After entering user 
information, the user saves them and can also edit them, if necessary. In this section, according to the user and system questions and answers, the appropriate treatment guide based on a set of ifthen rules, according to the patient's condition is provided by the system. Disease introduction, transmission, and prevention are included in the Education section of the app. The Contact with Physicians Section has the option of sending pictures of patients to physicians through SMS or email. Furthermore, the picture can be uploaded through a page in the application. The physician after the required investigation will respond to the patient. In the Useful Links section, by selecting a treatment center, the user can view the location and services information provided by treatment centers. In the final step, Quiz Satisfaction Survey Questionnaire v.7.0 was used to assess users' satisfaction.

\section{RESULTS}

This application was designed for informing and participation of patients in self-care. The sections of registration, education and prevention, user information, contact with physicians, disease information, and display of medical centers on the map were used in this application to execute the project.

\section{Designed prototype}

The features available in the application are as follows:

\section{Registration:}

In this section, the users register their information for login. After registration, they enter the application environment (Fig 1).

\section{User Information:}

In this section, the application receives users' information through the pages as shown in Figure 2. The information includes age, gender, job, number and location of lesions, etc. According to the information received, the application provides medical recommendations and essential tips for the user based on the information available in the database, and asks users to visit a treatment center (Fig 2).
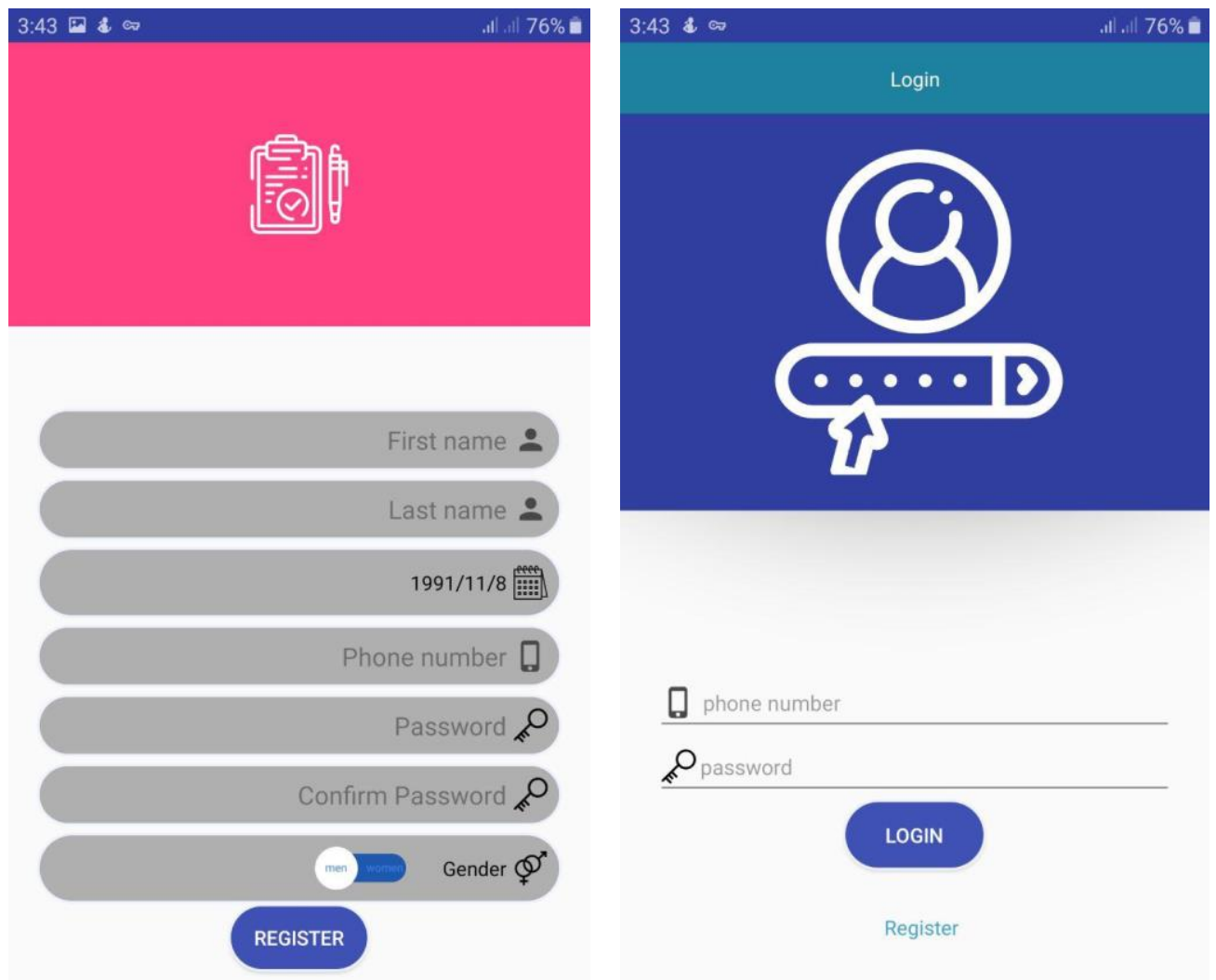

Fig 1: User registration 


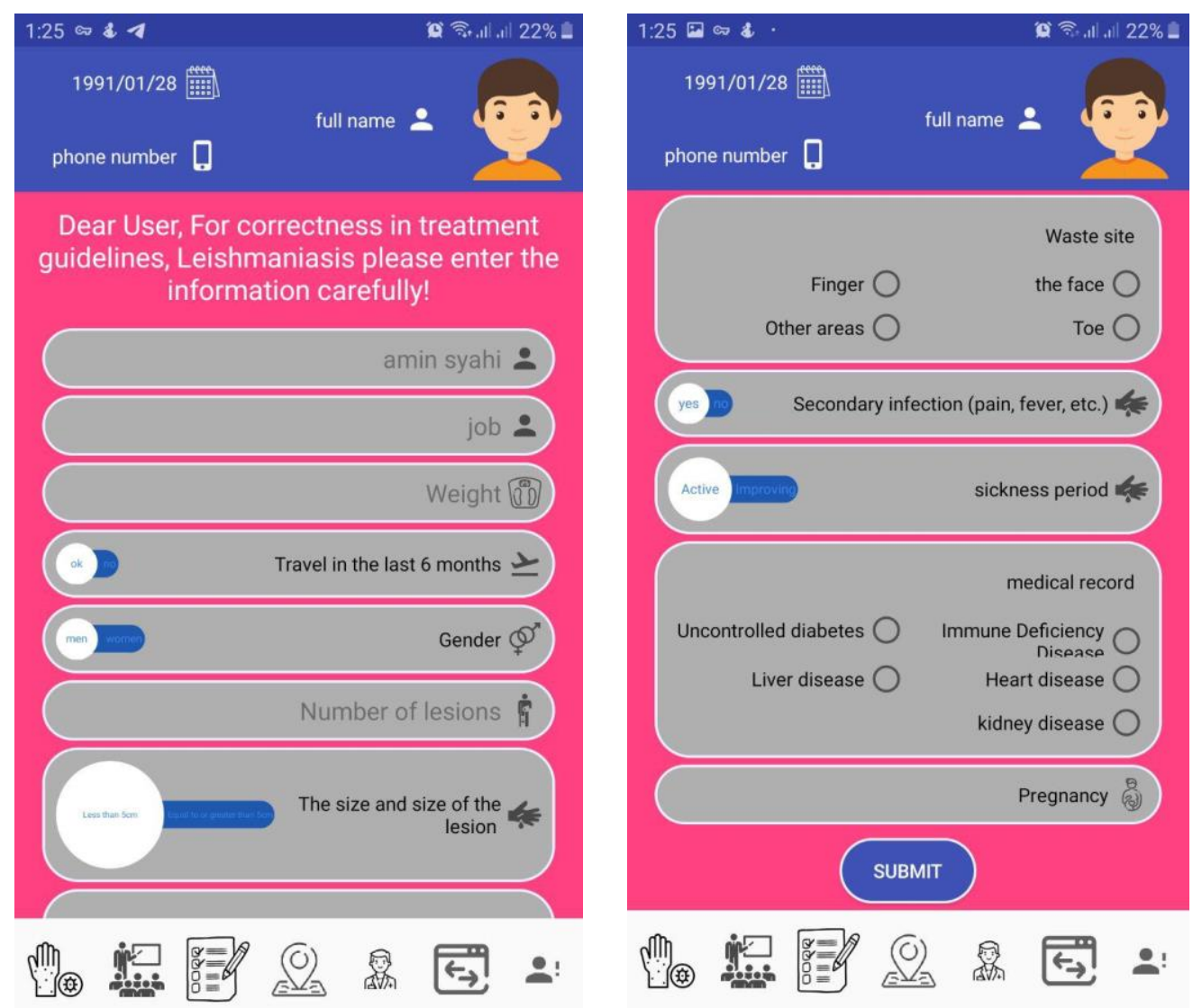

Fig 2: User information receiving

\section{Education and Prevention:}

Due to the impact of CL on quality of life of affected people, education and prevention lead to control and adherence to treatment. In this application, the Education section (Fig 3) included the definition of the disease and the importance and ways of transmission and prevention.

\section{Disease Information:}

This section of the application shows the prevalence and epidemiology of the disease in the world and in Iran (Fig 4). This information will be updated when new information about the education and prevention is published.

\section{Treatment Centers:}

In this part of the application, information of treatment centers are displayed according to the user's location on the map, so the user can contact the treatment center and also accessed the desired route through a navigation application (Google $\mathrm{Map}^{\mathrm{TM}}$ ) (Fig 5).

\section{Contact with Physicians:}

In this section, users can send a picture of the lesion and necessary explanations to their physicians (Fig
6).

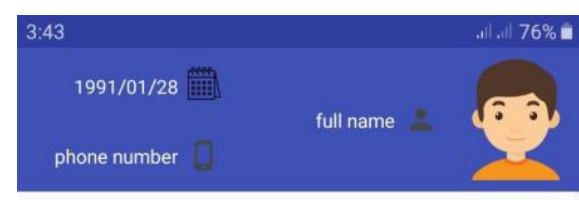

$$
\begin{aligned}
& \text { Training and prevention } \\
& \text { I.Training people }
\end{aligned}
$$

o Because of its small size, mosquitoes easily pass through the pores of the windows and doors of houses, so all pores in the doors and windows with sealants should be sealed and fine-grained nets should be used to prevent mosquitoes from entering.

o Using standard poison-infested mosquito nets or ordinary nets with very small pores during sleep is one of the best ways to prevent mosquito bites.

o The mosquito flight height is low at each flight but is also able to jump to the upper floors of the building. Therefore, all occupants of the upper floors of the building must also observe preventive safeguards.

o People are advised not to leave their homes as far as possible in endemic areas (sand, early mornings and possible in endemic areas (sand, early mornings and open insect repellent pens. To use.

o The mosquito carrier has a short oral appendix and is

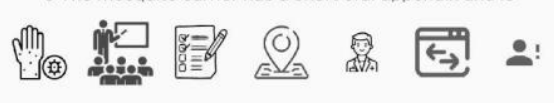

Fig 3: Education and prevention 


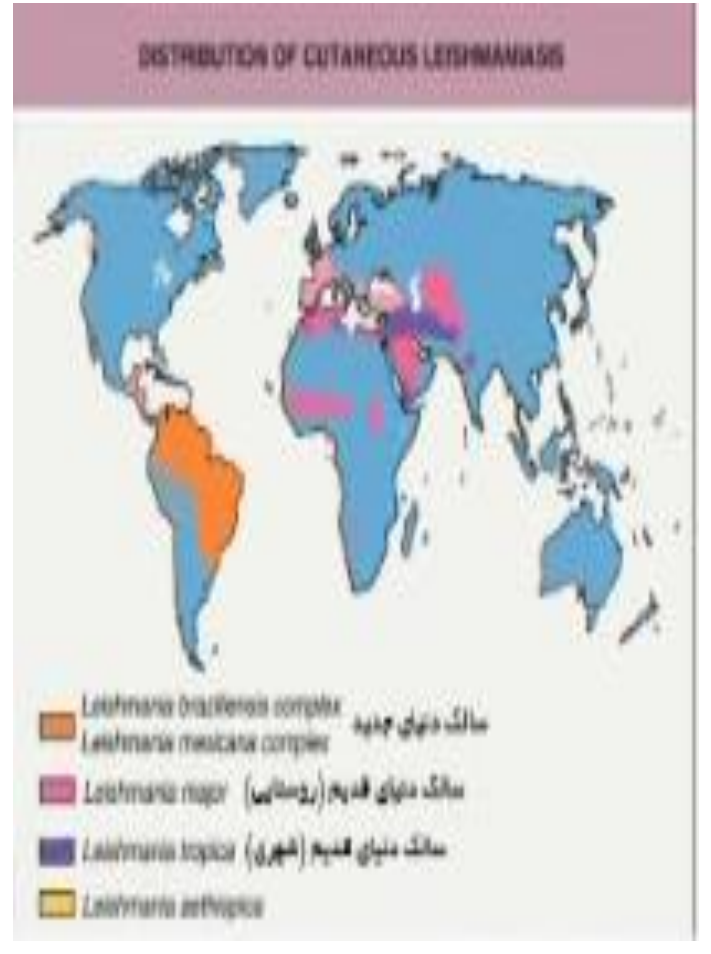

Fig 4: Prevalence status on the map

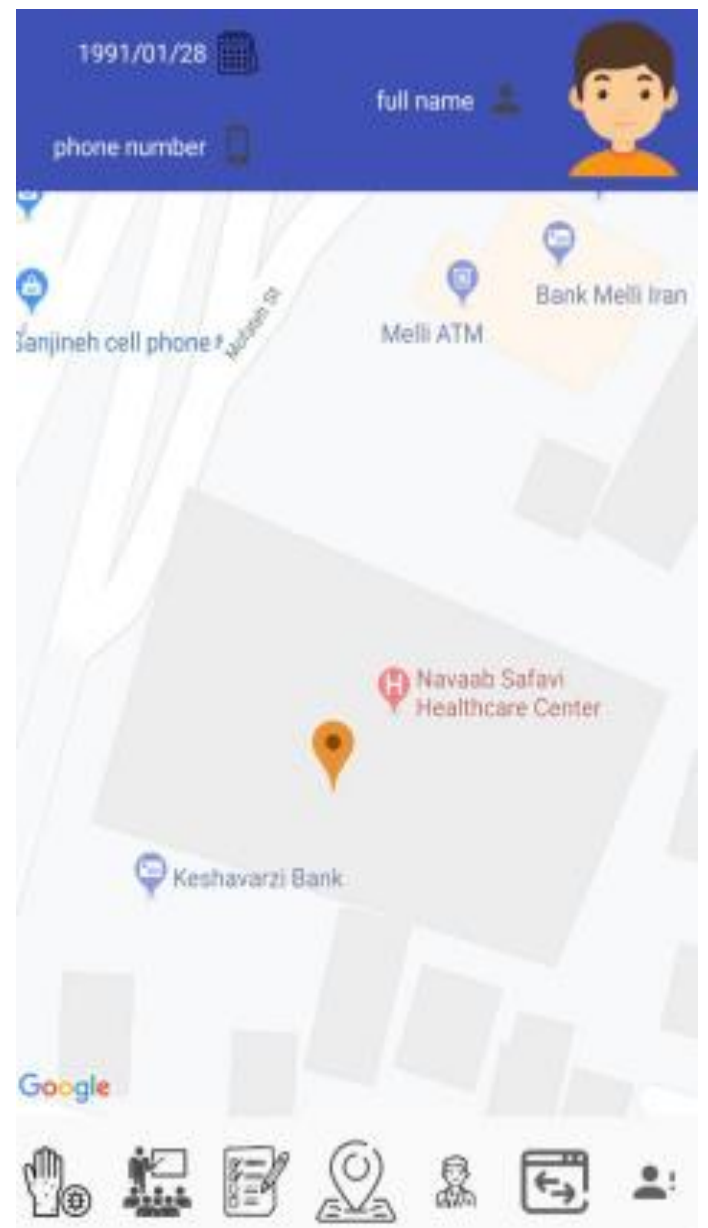

Fig 5: Useful links (address of treatment centers)

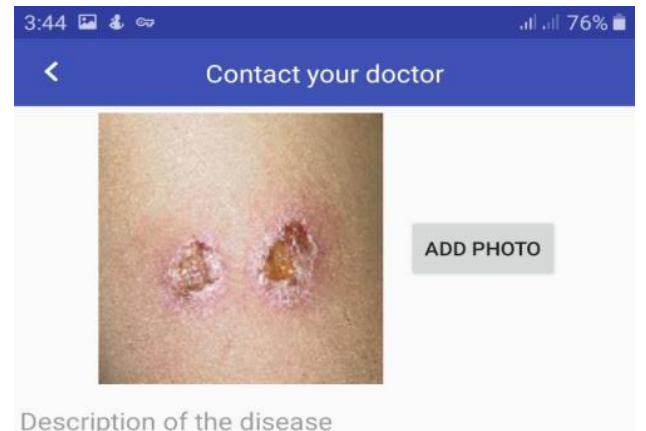

SEND

Fig 6: Contact with physicians

\section{Evaluation phase}

In the evaluation phase, the prototype application was provided to 10 patients referring to Shahid Babaei Hospital in Isfahan, Iran. The users in this stage were those with a positive diagnosis of CL. They were asked to use the application for a week and then their opinions were collected using a usability and user satisfaction survey questionnaire. A sample of questionnaire is shown in Appendix A. Data analysis results of the usability and user satisfaction survey questionnaire are presented in Table 1.

Table 1: Evaluation of patient satisfaction and usability of CL self-care application

\begin{tabular}{|l|c|c|c|}
\hline \multicolumn{1}{|c|}{ Variables } & $\begin{array}{c}\text { Total score of } \\
\text { each division }\end{array}$ & $\begin{array}{c}\text { Average } \\
\text { score }\end{array}$ & $\begin{array}{c}\text { Percentage } \\
\text { of } \\
\text { agreement }\end{array}$ \\
\hline $\begin{array}{l}\text { Working with } \\
\text { the system }\end{array}$ & 54 & 7.7 & 85.55 \\
\hline Screen & 36 & 8.17 & 90.77 \\
\hline $\begin{array}{l}\text { A collection of } \\
\text { terms and } \\
\text { information }\end{array}$ & 54 & 7.85 & 87.22 \\
\hline $\begin{array}{l}\text { System learning } \\
\text { capability }\end{array}$ & 54 & 7.9 & 87.77 \\
\hline $\begin{array}{l}\text { Total system } \\
\text { capability }\end{array}$ & 45 & 7.46 & 82.88 \\
\hline
\end{tabular}


According to Table 1, the mean scores of questionnaire domains of "your overall opinion about working with the system" (7.7), "display" (8.17), "terminology and information set" (7.85), "system learning ability" (7.9), and "system general abilities" (7.46) were in the range of 7-9 indicating that the application is evaluated at a proper level.

\section{DISCUSSION}

This study aimed to develop a mobile-based selfcare application for CL patients. After producing the final version of the application based on the users' comments, five sections (except registration part) were considered, each divided into sub-sections, which provided users with functional information in the form of text and image. In the present study, designing a mobile-based self-care application was considered as an effective step in patient' self-care and participation. This objective is in line with the results of a study by Bhunia et al. which listed telehealth features and capabilities and called it a powerful tool in CL control, especially in remote areas [16]. Bayatani \& Sadeghi suggested the use of geographic information system (GIS) technology for spatial analysis of environmental factors affecting CL to detect pathogens, finding patterns of their release into the environment, detecting their spatial aggregation and environmental factors exacerbating the disease for CL control and prevention management and planning [17]. They only prepared Health-GIS database.

The present study also considered the use of mHealth technology as a step towards disease management, patient self-care, and planning to reduce its consequences. In a study of Parmanto et al., the emphasis was on developing a mHealth system for self-care and skin problems management, supporting self-care tasks, monitoring patient status, skincare counseling, and bilateral communication between patients and physicians, which were in line with the objectives of the present study [18]. The purpose of this application was to provide educational information for self-care and CL patients' treatment follow-up that was provided to users as an Android-based application, which could be considered in line with a study by Mehdizadeh that used technology (a Teledermatology System) to facilitate communication with specialist physicians at any time and place and reduce physical transportation, and thereby increase access to skincare services for patients [19]. This application can be seen as a model for designing and developing similar systems and applications for treatment and care management and disease monitoring that aim to help control more and improve treatment quality and reduce consequences for patients. The only available system for recording CL information used in some centers is consistent with this self-care application in terms of having patient demographic information and disease symptoms; and the application is unique in having educational, connect with physicians and helpful links sections.

Considering the main objective of the present study to design and implement a mobile-based monitoring system for CL patients, and since addressing this and similar issues is one of the missions of the Ministry of Health at the national level, the findings of this study can be used for policy-making and planning in support of the design and implementation of a mobile-based monitoring system for CL patients by providing an electronic registration system and communications equipment for care centers' communication with patients to take a step towards controlling and managing the disease.

\section{CONCLUSION}

Mobile-based monitoring system for CL patients is a way that can eliminate some problems that patients and treatment staff face in the field of health and prevention of CL. The collaboration of health networks, CL treatment centers to accurately record patient information in the system and deliver timely educational information, reminders, and necessary messages can be effective in reducing disease consequences, recurrence, and patient costs. Ultimately, using the system will help prevent costs and provide timely information and education, send reminders to at-risk groups, and improve patients' current status and comfort.

\section{ACKNOWLEDGEMENT}

This paper was extracted from a dissertation approved at Tehran University of Medical Sciences under the code 98-01-31-41688, with the Ethics Code of IR.TUMS.SPH.REC.1397.103. The authors would like to express their gratitude to the Dermatology and Leprosy Center of Tehran University of Medical Sciences and Shahid Babaei Hospital in Isfahan, which participated in the various stages of this study.

\section{AUTHOR'S CONTRIBUTION}

K.N. and A.S. wrote the manuscript. K.N. compiled the data and L.S. and H.S. were supervisor and advisor. All the authors approved the final version of the manuscript.

\section{CONFLICTS OF INTEREST}

The authors declare no conflicts of interest regarding the publication of this study.

\section{FINANCIAL DISCLOSURE}

No financial interests related to the material of this manuscript have been declared. 


\section{REFERENCES}

1. Overdijkink SB, Velu AV, Rosman AN, vanBeukering MD, Kok M, Steegers-Theunissen RP. The usability and effectiveness of mobile health technology-based lifestyle and medical intervention apps supporting health care during pregnancy: Systematic review. JMIR mHealth and uHealth. 2018; 6(4): e109. PMID: 29691216 DOI: $10.2196 /$ mhealth.8834 [ubMed]

2. Liravi MM, Shahbahrami A. Priority of mobile health applications and its infrastructures. Journal of Information Technology Management. 2015; 7(2): 407-28.

3. Mehdipour Y, Khammarnia M, Karimi A, Alipour J, Seyedzaei F, Ebrahimi S. Effective factors in acceptance of mobile health from physicians point of view. Journal of Modern Medical Information Sciences. 2017; 3(1): 30-7.

4. Pohl M. 325,000 mobile health apps available in 2017 - Android now the leading mHealth platform [Internet]. 2017 [cited: 3 Jan 2020]. Available at: https://research2guidance.com/325000-mobilehealth-apps-available-in-2017.

5. Jahns RG. The mHealth app market will grow by $15 \%$ to reach $\$ 31$ billion by 2020 [Internet]. 2017 [cited: 3 Jan 2020]. Available at: https://research2guidance.com/the-mhealth-appmarket-reaches-31-billion-by-2020.

6. Yasini M, Beranger J, Desmarais P, Perez L, Marchand G. mHealth quality: A process to seal the qualified mobile health apps. Stud Health Technol Inform. 2016; 228: 205-9. PMID: 27577372 [PubMed]

7. Molaei K, Ahmadi M. The role of mobile health apps to facilitate self-care. Journal of Modern Medical Information Sciences. 2017; 3(1): 44-55.

8. Moghaddasi H, Mehdizadeh H. Mobile health for diagnosis and management of skin lesions. Journal of Health and Biomedical Informatics. 2016; 3(2): 15565.

9. Ershad Sarabi R, Sadoughi F, Jamshidi Orak R, Bahaadinbeigy K. Role of mobile technology in Iran healthcare system: A review study. Journal of Health and Biomedical Informatics. 2018; 4(4): 313-26.

10. Noorpisheh S, Naghizadeh MM, Nikrouz L. A study on the life quality of patients suffering from leishmaniasis. Journal of Fasa University of Medical Sciences. 2013; 3(2): 155-62.

11. Smith M, Saunders R, Stuckhardt L. Best care at lower cost: The path to continuously learning health care in America. Washington (DC): National Academies Press (US); 2013.

12. World Health Organization. Manual for case management of cutaneous leishmaniasis in WHO Eastern Mediterranean Region, WHO Regional Publications, Eastern Mediterranean Series. 2011.

13. Anderson K, Burford O, Emmerton L. Mobile health apps to facilitate self-Care: A qualitative study of user experiences. PLoS One. 2016; 11(5): e0156164. PMID: 27214203 DOI: 10.1371/journal.pone.0156164 [PubMed]

14. Safdari R, Shahmoradi L, Garavand A, Aslani N, Valipour A, Bostan H. Design and evaluation of a mobile-based application for patients with type 2 diabetes: Case study of Shariati hospital in Tehran, Iran. Hospital Practices and Research. 2018; 3(4): 130-6.

15. Sheikh Taheri A, Norouzi E, Sadoughi F. Developing a mobile-based self-care application for patients with breast cancer undergoing chemotherapy. Journal of Health Administration. 2019; 22(4): 35-49.

16. Bhunia GS, Kesari S, Chatterjee N, Kumar V, Das P. Telehealth: A perspective approach for visceral leishmaniasis (kala-azar) control in India. Pathog Glob Health. 2012; 106(3): 150-8. PMID: 23265372 DOI: 10.1179/2047773212Y.0000000014 [PubMed]

17. Bayatani A, Sadeghi A. Spatial analysis of effective environmental factors on Cutaneous leishmaniasis In Iran using GIS. A report to Ministry of Health and Medical Education: Iran, Tehran. 2009.

18. Parmanto B, Pramana G, Daihua XY, Fairman AD, Dicianno BE. Development of mHealth system for supporting self-management and remote consultation of skincare. BMC Med Inform Decis Mak. 2015; 15: 1-8. PMID: 26714452 DOI: $10.1186 / \mathrm{s} 12911-015-0237-4$ [PubMed]

19. Mehdizadeh H, Ayatollahi H, Esmaeili N, Kamkar Haghighi M. Designing and building a teledermatology system. Journal of Mazandaran University of Medical Sciences. 2015; 25(123): 17084. 